\title{
Operationalising an ensemble approach in the description of uncertainty in atmospheric dispersion modelling and an emergency response
}

\author{
P. Bedwell ${ }^{1, *}$, I. Korsakissok ${ }^{2}$, S. Leadbetter ${ }^{3}$, R. Périllat ${ }^{2,4}$, Cs. Rudas ${ }^{5}$, J. Tomas $^{6}$, J. Wellings ${ }^{1}$, \\ G. Geertsema ${ }^{7}$ and H. de Vries ${ }^{7}$ \\ ${ }^{1}$ PHE - Public Health England, Chilton, Didcot, UK. \\ ${ }^{2}$ IRSN - Institut de Radioprotection et de Sûreté Nucléaire, Fontenay-aux-Roses, France. \\ 3 Met Office, Exeter, UK. \\ ${ }^{4}$ PHIMECA Engineering, Paris, France. \\ 5 EK - Centre for Energy Research, Budapest, Hungary. \\ ${ }^{6}$ RIVM - National Institute for Public Health and the Environment, Bilthoven, The Netherlands. \\ ${ }^{7}$ KNMI - Royal Netherlands Meteorological Institute, De Bilt, The Netherlands.
}

\begin{abstract}
Uncertainties were propagated through the chain of atmospheric dispersion and radiological assessment models based on an ensemble approach for a range of scenarios. It was apparent that the time taken to complete model runs, ranging from several hours to a few tens of hours, was not appropriate for an emergency response. Thus, for an operational method, there was a requirement to reduce the number of ensemble members and/or reduce model run time for a single ensemble member, such that a measure of uncertainty may be obtained within the timeframe of one hour, but without significant detriment to the model endpoints derived, the uncertainty estimated and the radiation protection advice inferred. This study proposes recommendations for operationalising an ensemble approach used in the description of uncertainty in atmospheric dispersion modelling and an emergency response.
\end{abstract}

Keywords: operational / ensemble / uncertainty / atmospheric dispersion / emergency response

\section{Introduction}

Within the European project CONFIDENCE, Work Package 1 (WP1) focused on uncertainty in the pre-release and early phase of a radiological emergency. Uncertainty tends to be greatest during the pre-release phase, in part because measurements are not yet available. This is in contrast to the post-release phase, when measurements can be used to reduce model uncertainty (Lahtinen et al., 2010; Bleher et al., 2020).

Mathieu et al. (2018) presented guidelines ranking uncertainties for atmospheric dispersion modelling. These guidelines introduced the concept of the application of ensembles in the description of atmospheric dispersion model uncertainty and provided estimates of ranges and distributions of uncertainties on atmospheric dispersion model input parameters. By way of a range of scenarios (including hypothetical scenarios), these uncertainties were propagated through the chain of atmospheric dispersion and radiological

*Corresponding author: peter. bedwell@phe.gov.uk assessment models based on an ensemble approach (Berge et al., 2019; Korsakissok et al., 2019b). The time taken to complete model runs ranged from several hours to a few tens of hours. These times are too long for practical use in an emergency. An operational method would need to obtain a measure of uncertainty within approximately one hour. To achieve that, the number of ensemble members must be reduced and/or model run time for a single ensemble member must be reduced. This would need to be achieved without significant detriment to model endpoints derived, uncertainty estimated and radiation protection advice inferred. A more detailed description of the work performed can be found in Bedwell et al. (2019).

\section{Reducing run time for a single ensemble member}

Hypothetical scenarios described by Korsakissok et al. (2019b) were re-run using an ensemble approach and modified model set-up. Modelling schemes, model domain and output 
resolution were each modified. The modifications of the modelling schemes involved reducing the number of puffs or particles and the frequency of the puff/particle calculations. Two atmospheric dispersion models were considered: a Gaussian Puff model implemented in the SINAC programme system developed by the Hungarian Centre for Energy Research (MTA EK), and the UK Met Office's Lagrangian Particle model, NAME.

The most computationally expensive aspects of the dispersion modelling procedure were the calculation of model endpoints and writing of results for the Gaussian Puff approach, and the processing of model particle loops for the Lagrangian Particle approach. Although the computationally expensive aspects differed between the two models, model run time could be reduced by the same methods; by reducing the number of model puffs or particles and by reducing the size of the model domain. The implementation of such methods for reducing model run time did not result in significant differences in model output when compared to previously performed runs (on the basis of a full model set-up configuration), and therefore the representation of uncertainty was maintained.

The optimum size of the model domain can be estimated a priori by performing a scoping run (using a simple straight line Gaussian plume model and/or trajectory model), estimating the likely distance of interest, based on conservative meteorological conditions and release size (inferred from the meteorological and source term ensemble, respectively). Furthermore, assessments may initially focus on key model endpoints, which are likely to be those used in the provision of protective action (rather than food restriction) advice, and which are likely to require the consideration of a smaller domain.

Reducing the temporal resolution of model output resulted in only moderate computational savings, but this could still be worthwhile in an emergency response. Reducing the number of modelled radionuclides can also be beneficial. This can be achieved by modelling a unit release of a small number of pseudo radionuclides, where each is representative of the chemical and physical characteristics of a group of radionuclides. An alternative approach would be to model radionuclides explicitly, but to reduce the number considered to only those which contribute (in order of ranking) a predefined percentage of the dose. The dose could then be scaled accordingly after completion of the full dispersion calculation.

Note that additional computational resource will speed up the processing of a large number of ensemble model runs and reduce computational time, but at a financial cost. However, consideration of the utilisation of current computational resource should also be made, by way of launching multiple simulations in parallel on different cores of a computer or on different computers.

\section{Optimising the number of ensemble members used in the simulations}

Reducing the number of meteorological and/or source term ensemble members can reduce run time, but an optimum number of members must remain to accurately represent the underlying uncertainty. It can be a significant challenge to identify which members of these input ensembles are most important to retain, particularly when prior knowledge of their impact on dispersion model output is limited. This can be addressed by clustering ensemble members that are deemed "similar" with respect to well-defined criteria. Clustering of a meteorological ensemble with respect to wind direction is considered below. Automated methods, based on mathematical criteria, have also been tested on an ensemble of source terms (Bedwell et al., 2019).

\subsection{Clustering meteorological ensembles}

Hypothetical scenarios described by Korsakissok et al. (2019b) were re-run, this time using a clustered ensemble. The results and run times were compared with original runs (based on the full configuration of ensemble members). The Royal Netherlands Meteorological Institute (KNMI) provided meteorological ensemble data based on the non-hydrostatic convection-permitting HARMONIE-AROME weather model (Bengtsson et al., 2017). The atmospheric dispersion model NPK-PUFF, developed by the Netherlands Institute for Public Health and the Environment (RIVM) was used to generate the results.

A fast and simple approach is to consider a meteorological variable that is (in general) most influential in determining key endpoints; wind direction at the release location was considered here. This proposed method includes the assessment of wind direction meteograms in order to cluster ensemble members before performing the atmospheric dispersion modelling.

The "level of agreement" (reflecting relative likelihood and not absolute probability) between dispersion simulation members used to derive time integrated activity concentrations in air (TIACs) is shown in Figure 1. A reduction in dispersion ensemble members was achieved by considering a reduced number of meteorological members, which in turn was determined where agreement in wind direction between the ensemble members was observed and where uniformity in wind direction as a function of time for any one ensemble member was observed, over the potential release period. This approach has been presented in Tomas and Twenhöfel (2019). The reduction from a 650 member ensemble to a 380 and 280 member ensemble had little effect on the description of uncertainty. However, reducing the number of dispersion simulation members to 135 did affect the outcome, and the spatial spread was less well captured. Such reductions in the number of dispersion simulation members made significant run time savings. Although run time was not reduced to one hour, this could have been achieved, for example by reducing the number of release heights considered.

Clustering based on wind direction alone provides a useful example, but has been demonstrated for only one scenario here. A much wider range of scenarios would need to be considered in order to identify the most suitable clustering method and criteria. Wind direction should probably always be included in clustering criteria, but other meteorological variables may also be required, depending on the scenario. These might include wind speed, boundary layer height, atmospheric stability and precipitation.

In addition to the use of meteograms, it is recommended to cluster on the basis of model output from sets of atmospheric 

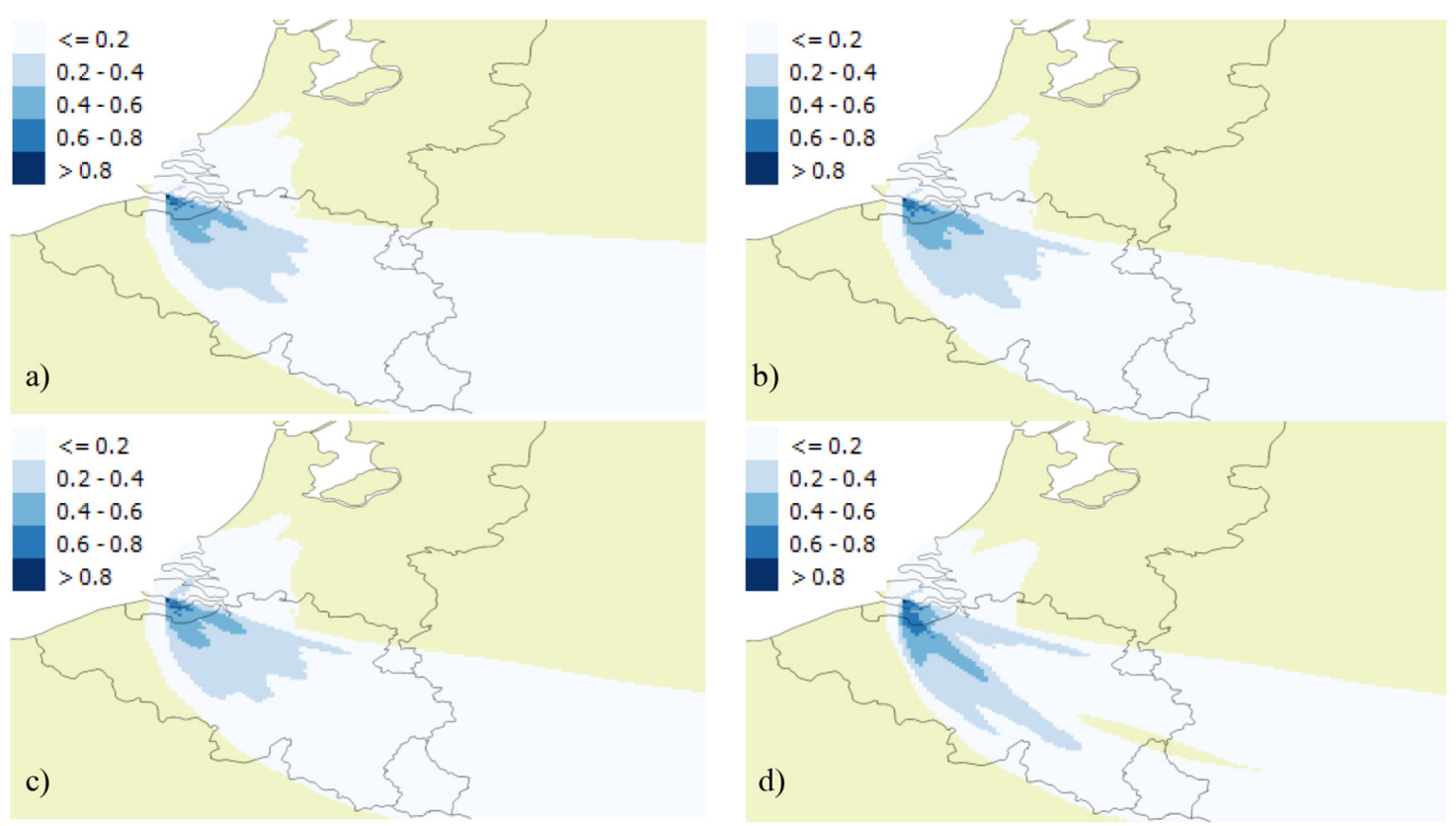

Fig. 1. Level of agreement (where $0=$ no agreement and $1=$ complete agreement) between results exceeding $5000 \mathrm{~Bq} \mathrm{~h} \mathrm{~m}^{-3} \mathrm{TIAC}$ of ${ }^{131} \mathrm{I}$ for $(\mathrm{a})$ a full ensemble (650 members); (b) a reduced 380 member ensemble; (c) a reduced 280 member ensemble; and (d) a reduced 135 member ensemble.

dispersion model runs performed in a very simplified mode, for example using particle trajectories, only using single site meteorological conditions, using a different and simpler model, and introducing significant simplifications such as removing the assessment of deposition for scenarios where there exists large confidence in no precipitation (where the latter will only be suitable for advice in respect of certain protective actions).

\subsection{Application of sampling methods}

Sampling can also be used to reduce the number of ensemble members. In this study, a Monte Carlo ensemble simulation was performed by randomly sampling meteorology and source terms, and thereby significantly reducing the number of model runs without any detriment to the results obtained. A method to verify if a suitable number of simulations has been chosen is to analyse the ensemble results following each addition of a further simulation; the optimum number of simulations will be reached when the model results sufficiently converge. It is clear in Figure 2 that fewer simulations are required to derive a stable ensemble mean and median compared to the ensemble maximum and that the description of uncertainty for all considered statistical endpoints (barring the ensemble maximum) does not change significantly beyond approximately 40 simulations.

\section{Uncertainty representation for operational purposes}

Uncertainty could be presented by producing tables of values of key endpoints such as area/number of people affected, or maximum distance above a given threshold dose or environmental concentration. However, this may fail to communicate important information and can even be misleading. For example, the magnitudes of the areas, numbers of people and maximum distances can be similar, but the spatial coverage may be very different.

Uncertainty could be presented using figure of merit in space, FMS. This concept provides an indication of the level of agreement between ensemble members, as a result of the degree of overlap between zones of threshold exceedance predicted by different simulations. This could be extended to figure of merit of population which may be of value for radiation protection advice. A limitation is that two sets of ensemble results of threshold exceedance may have the same FMS, but may differ in other significant ways. For example, results that are similar in shape but are shifted in space may have the same FMS as results that differ significantly in shape but are shifted relatively little in space.

In addition, ratios of high and low percentiles (90th/10th or $75 \mathrm{th} / 25 \mathrm{th}$ ), as proposed by Hamburger et al. (2019) and Berge et al. (2019), are a useful indicator of scalar uncertainty, for example when considering the dose received at a single location.

Therefore, where only numerical values are derived, the production of FMS, and one or more of the area and/or number of people affected and the maximum distance (above a given threshold), and potentially percentile ratios, are recommended. The temporal evolution of uncertainties may also be of significant value and warrant consideration.

In view of the aforementioned limitations, the preferred option is to present numerical values together with a graphical representation of uncertainty. This could be achieved by using level of agreement plots (e.g. Fig. 1) and percentile plots. Model ensemble output should be presented in terms of likelihood (or agreement) maps and not probability maps. 


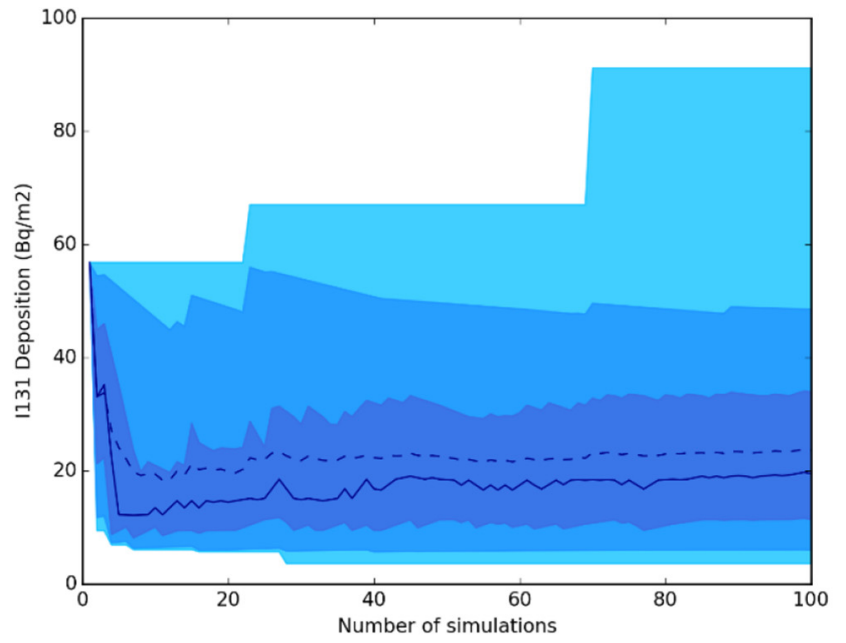

(a)

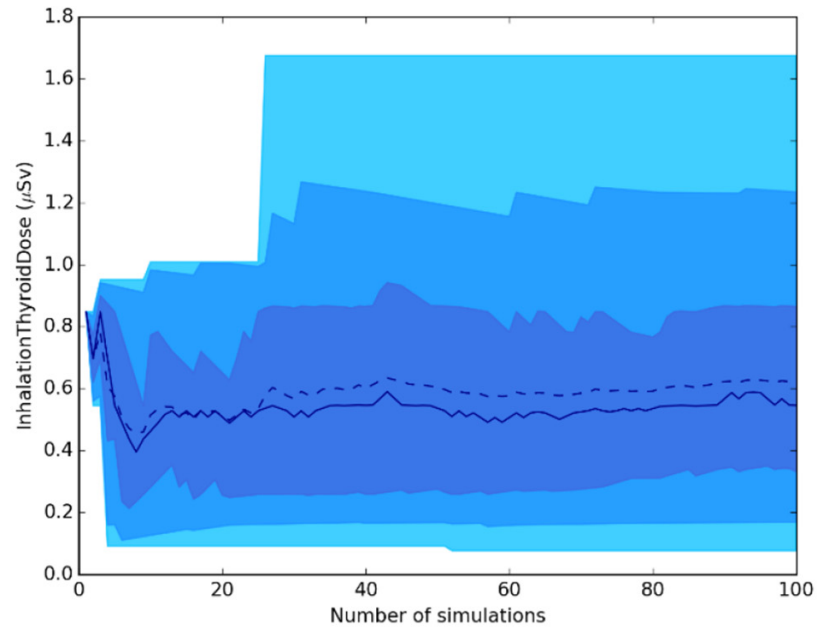

(b)

Fig. 2. (a) ${ }^{131}$ I deposition concentration and (b) inhalation thyroid dose (spatially) averaged over the model domain and integrated to $24 \mathrm{~h}$ after the start of the release, for varying numbers of simulations using a Monte Carlo sampling method. Ensemble mean $=$ dashed line. Ensemble median $=$ solid line. Region of lightest shading $=0$ th to 100 th percentile range. Region of darker shading $=20$ th to 80 th percentile range. Region of darkest shading $=40$ th to 60 th percentile range.

\section{Recommendations}

Recommendation 1: It is recommended that uncertainties in atmospheric dispersion simulations for emergency response are accounted for by way of an ensemble approach. Such an approach should include the primary uncertainties, notably meteorological and source term uncertainties.

Two approaches were investigated in this study: (1) reducing the model run time for a single ensemble member and (2) reducing the number of ensemble members. Of these two approaches, the former should be prioritised. Approach (2) may not be necessary, but could be beneficial in circumstances where the full ensemble has a large number of members.

Recommendation 2: It is recommended that efficiency savings in model set-up are identified and implemented to reduce model run time for a single ensemble member (without significant detriment to model endpoints derived, uncertainty estimated and radiation protection advice inferred). The greatest benefit is most likely to result from: (a) reducing the number of model puffs or particles or, in the case of an Eulerian model, using a coarser resolution; and (b) reducing the size of the model domain.

Recommendation 3: It is recommended to optimise the number of ensemble members so that the ensemble is still representative of the full uncertainty. Two approaches may be explored to achieve this goal (and may be used together):

- clustering the (meteorological and/or source term) ensem-

ble members, to select members that are representative of a subset of the ensemble;

- using sampling methods, to avoid running dispersion calculations for all different possibilities (especially when several sources of uncertainty are taken into account).

Recommendation 4: It is recommended to work on advanced clustering methods with meteorological offices responsible for providing the data in case of an emergency, so that the particular needs for this application are well understood by data providers; if possible, tailor-made ensembles should be specifically designed. Clustering methods should ideally consider dispersion model output variables and not only meteorological variables.

Recommendation 5: It is recommended that a range of uncertainty indicators are considered. Such uncertainty indicators should include a combination of tabulated numerical values and graphical representations. Uncertainty indicators in the form of numerical values may include: surface area and number of people affected; maximum distance above a given threshold dose or environmental concentration; figure of merit in space (FMS) and population (FMP). Uncertainty indicators in graphical form may include level of agreement plots and percentile plots. A spread of uncertainty indicators and presentation methods is vital to fully understand model endpoint uncertainties which are spatially and temporally complex.

\section{Future work}

A methodology should be developed for simplifying ensemble model runs, focusing on reducing the time taken to process and output model results. Different spatial and temporal resolutions of meteorological data could be considered in an investigation of the effect on the accuracy of results and computational time. The use of an emulator to save computational time could be investigated. It would be beneficial to investigate the inclusion of other meteorological variables (such as wind speed, boundary layer height, atmospheric stability and precipitation) as part of any clustering criteria. Further work assessing the ability of different methods to suitably sample ensemble members from a full ensemble configuration would be of value (e.g. adaptive sampling and Latin Hypercube Methods). Investigation into whether the work of Klonner (2013) and Galmarini et al. (2004a, 2004b and 2004c) could be applied to the estimation of uncertainty within the provision of radiological protection advice following an accidental release to atmosphere would also be worthwhile. Further work is required to assess the 
suitability of a priori clustering on the basis of statistical analysis. The work already carried out in relation to evaluating uncertainty estimation by comparison with environmental observations (Korsakissok et al., 2019a) should be extended. Furthermore, efficient methods should be developed for combining prior knowledge of uncertainties with observational data. A more detailed description of proposed future work can be found in Bedwell et al. (2019).

Acknowledgement. The work described in this paper was conducted within the CONFIDENCE project which was part of the CONCERT project. This project has received funding from the Euratom research and training programme 20142018 under grant agreement No. 662287.

Disclaimer (Art. 29.5 GA). This publication reflects only the author's view. Responsibility for the information and views expressed therein lies entirely with the authors. The European Commission is not responsible for any use that may be made of the information it contains.

\section{References}

Bedwell P, Korsakissok I, Leadbetter S, Périllat R, Rudas C, Tomas J, Wellings J. 2019. Guidelines for the use of ensembles in the description of uncertainty in atmospheric dispersion modelling: operational applications in the context of an emergency response. CONCERT Deliverable D9.5. Available from https://concerth2020.eu/en/Publications.

Bengtsson L, Andrea U, Aspelien T, Batrak Y, Calvo J, de Rooy W, Gleeson E, Hansen-Sass B, Homleid M, Hortal M, Ivarsson K, Lenderink G, Niemela S, Pagh Nielsen K, Onvlee J, Rontu L, Samuelsson P, Santos Munoz D, Subias A, Tijm S, Toll V, Yang X, Ødegaard Koltzow M. 2017. The HARMONIE-AROME model configuration in the ALADIN-HIRLAM NWP system. Mon. Weather Rev. https://doi.org/10.1175/MWR-D-16-0417.1.

Berge E, Klein H, Ulimoen M, Andronopoulos S, Lind O-C, Salbu B, Syed N. 2019. Guidelines for the use of ensemble calculations in an operational context, indicators to assess the quality of uncertainty modeling and ensemble calculations, and tools for ensemble calculation for use in emergency response, D9.5.2 Ensemble calculation for hypothetical accident scenarios in Europe: the Norway case study. CONCERT Deliverable D9.5. Available from https://concert-h2020.eu/en/Publications.

Bleher M, Gering F, Stöhlker U, Karhunen T, Nalbandyan-Schwarz A, Woda C, Mafodda A. 2020. Reduction of uncertainties in exposure assessments based on environmental monitoring data. Radioprotection 55(HS1): https://doi.org/10.1051/radiopro/2020016.

Galmarini S, Bianconi R, Addis R, Andronopoulos S, Astrup P, Bartzis J, Bellasio R, Buckley R, Champion H, Chino M, D'Amours R, Davakis E, Eleveld H, Glaab H, Manning A, Mikkelsen T, Pechinger U, Polreich E, Prodanova M, Slaper H, Syrakov D, Terada H, Van der Auwera L. 2004a. Ensemble dispersion forecasting-Part II: application and evaluation. Atmos. Environ. 38(28): 4619-4632.

Galmarini S, Bianconi R, Klug W, Mikkelsen T, Addis R, Andronopoulos S, Astrup P, Baklanov A, Bartniki J, Bartzis J, Bellasio R, Bompay F, Buckley R, Bouzom M, Champion H, D'Amours R, Davakis E, Eleveld H, Geertsema G, Glaab H,
Kollax M, Ilvonen M, Manning A, Pechinger U, Persson C, Polreich E, Potemski S, Prodanova M, Saltbones J, Slaper H, Sofiev M, Syrakov D, Sorensen J, Van der Auwera L, Valkama I, Zelazny R. 2004b. Can the confidence in long range atmospheric transport models be increased? The pan-european experience of ensemble. Radiat. Prot. Dosim. 109(1-2): 19-24.

Galmarini S, Bianconi R, Klug W, Mikkelsen T, Addis R, Andronopoulos S, Astrup P, Baklanov A, Bartniki J, Bartzis J, Bellasio R, Bompay F, Buckley R, Bouzom M, Champion H, D’Amours R, Davakis E, Eleveld H, Geertsema G, Glaab H, Kollax M, Ilvonen M, Manning A, Pechinger U, Persson C, Polreich E, Potemski S, Prodanova M, Saltbones J, Slaper H, Sofiev M, Syrakov D, Sorensen J, Van der Auwera L, Valkama I, Zelazny R. 2004c. Ensemble dispersion forecasting-Part I: concept, approach and indicators. Atmos. Environ. 38(28): 4607-4617.

Hamburger T, Gering F, Ievdin I, Schantz S. 2019. Guidelines for the use of ensemble calculations in an operational context, indicators to assess the quality of uncertainty modeling and ensemble calculations, and tools for ensemble calculation for use in emergency response, D9.5.4 Uncertainty propagation through a terrestrial food chain and dose model. CONCERT Deliverable D9.5. Available from https://concert-h2020.eu/en/Publications.

Klonner R. 2013. Clustering ECMWF ENS ensemble predictions to optimise FLEXPART plume dispersion ensembles. Master's Thesis, University of Vienna.

Korsakissok I, Périllat R, Andronopoulos S, Astrup P, Bedwell P, Berge E, Quérel A, Klein H, Leadbetter S, Saunier O, Sogachev A, Tomas J, Ulimoen M. 2019a. Guidelines for the use of ensemble calculations in an operational context, indicators to assess the quality of uncertainty modeling and ensemble calculations, and tools for ensemble calculation for use in emergency response, D9.5.3 Ensemble calculation for a past accident scenario: the Fukushima case study. CONCERT Deliverable D9.5. Available from https://concert-h2020.eu/en/Publications.

Korsakissok I, Périllat R, Geertsema G, De Vries H, Hamburger T, Tomas J, Andronopoulos S, Bedwell P, Charnock T, Ievdin I, Leadbetter S, Pazmandi T, Rudas C, Scheele R, Sogachev A, Szanto P, Wellings J. 2019b. Guidelines for the use of ensemble calculations in an operational context, indicators to assess the quality of uncertainty modeling and ensemble calculations, and tools for ensemble calculation for use in emergency response, D9.5.1 Ensemble calculations for the atmospheric dispersion of radionuclides. Hypothetical accident scenarios in Europe: the REM case studies. CONCERT Deliverable D9.5. Available from https://concert-h2020.eu/en/Publications.

Lahtinen J, Aage H, Ammann M. 2010. Guidance on monitoring and data assimilation, Radioprotection 45(5): S161-S169.

Mathieu A, Korsakissok I, Périllat R, Chevalier-Jabet K, Stephani F, Fougerolle S, Créach V, Cogez E, Bedwell P. 2018. Guidelines ranking uncertainties for atmospheric dispersion, D9.1.3 Guidelines describing source term uncertainties. CONCERT Deliverable D9.1. Available from https://concert-h2020.eu/en/Publications.

Tomas J, Twenhöfel C. 2019. The effects of uncertainties on the radiological assessment in the (pre-) release phase of a nuclear accident. In: 19th International Conference on Harmonisation within Atmospheric Dispersion Modelling for Regulatory Purposes, Bruges, Belgium.

Cite this article as: Bedwell P, Korsakissok I, Leadbetter S, Périllat R, Rudas C, Tomas J, Wellings J, Geertsema G, de Vries H. 2020. Operationalising an ensemble approach in the description of uncertainty in atmospheric dispersion modelling and an emergency response. Radioprotection 55(HS1): S75-S79 